\title{
Conduite à tenir devant une image radioclaire des mâchoires
}

\section{Jaw radiolucent lesions}

\section{Résumé}

\section{MOTS-CLEFS :}

- Lésions radioclaires, radiologie, mâchoires, maxillaire,

mandibule, os

\section{KEYWORDS:}

- Lesions, imaging, radiolucent, jaws, maxillar. mandibular bone
Les lésions ostéolytiques des mâchoires sont fréquentes et se manifestent souvent par une clarté radiologique. Elles peuvent alors correspondre ò des kystes (odontogènes inflammatoires, dysembryonnaires dentaires ou dysembryonnaires osseux) ou à des tumeurs bénignes ou malignes, d'origine dentaire ou non. Seuls l'interrogatoire, un examen clinique bien conduit et l'expérience du praticien permettent de s'orienter vers l'une ou l'autre de ces possibilités diagnostiques et d'opter ainsi pour une prise en charge thérapeutique optimale.

\section{Abstract}

Jaws osteolytic lesions are frequent and often lead to a radiolucent lesion. They can then correspond to cysts or tumors. On one side, cysts can be whether of infectious dental origin or of dental and osseous dysembryogenic origin. On the other side, tumors can be benign or malign, odontogenic or not. Clinical step is primordial to allow the experimented practitioner to shape the diagnosis and choose the best therapeutic solution.
AOS 2014;270:4-12 DOI: $10.1051 / \mathrm{aOs} / 2014402$ (C) EDP Sciences 2014

Blandine RUHIN-PONCET
MD, PhD, HDR - Chirurgien Maxillofacial, Stomatologue,
Pathologies osseuses des mâchoires
Service de stomatologie et chirurgie maxillo-faciale
Groupe hospitalier Pitié Salpêtrière - 47, boulevard de l'Hôpital 75013 Paris
Nadine MARTIN-DUVERNEUIL
Service de neuroradiologie
Groupe hospitalier Pitié Salpêtrière - 47, boulevard de l'Hôpital 75013 Paris




\section{INTRODUCTION}

Les images radioclaires des mâchoires sont des lésions fréquentes volontiers considérées comme banales. Elles recouvrent cependant des étiologies multiples au pronostic variable, nécessitant une attitude thérapeutique et une surveillance adaptées. La découverte d'une lésion ostéolytique doit donc bénéficier aujourd'hui d'une approche diagnostique optimale permettant son évaluation la plus précise possible. Celle-ci repose avant tout sur la confrontation des données clinico-radiologiques et à l'heure des progrès et de la valeur des technologies avancées, il est primordial de rappeler le caractère essentiel d'un interrogatoire et d'un examen clinique bien poussé, point de départ obligatoire d'une pré-orientation diagnostique de qualité.

Le plus souvent, quelle que soit la symptomatologie d'appel, l'examen clinique débouchera sur une demande d'examen radiologique complémentaire, qui reste le meilleur procédé pour cerner le diagnostic avant l'incontournable prise en charge chirurgicale et la conclusion anatomopathologique. Celui-ci devra être orienté par le siège de la lésion, son caractère unique ou multiple... Actuellement, l'examen de base, facilement accessible, reste lorthopantomogramme ou cliché panoramique dentaire, qui permet avec une image globale des mâchoires une première analyse de la lésion mais aussi le dépistage d’autres images, et donc de lésions, jusque-là insoupçonnées. Il ne représente cependant le plus souvent qu'une étape, et il est essentiel d'en connaître les avantages et surtout les limites pour orienter de façon adaptée la suite des investigations.

\section{SITUATIONS CLINIQUES}

Plus concrètement, on peut se retrouver devant trois situations cliniques :

$\checkmark 1^{\text {re }}$ situation : le patient a des doléances particulières concernant la sphère oromaxillofaciale (tuméfaction, douleur, déplacement ou mobilité dentaire, écoulement nauséabond). L'examen clinique bien conduit fait envisager un bilan radiographique de type panoramique dentaire qui découvre une image radioclaire sur le maxillaire ou la mandibule ;

$12^{\mathrm{e}}$ situation : le patient est allé récemment consulter un dentiste ou un stomatologue, et le cliché panoramique dentaire réalisé lors de cette consultation atteste la présence d'une image radioclaire des mâchoires, ainsi de découverte fortuite ;

$13^{\text {e }}$ situation : le bilan radiologique a été demandé dans le cadre d'un contrôle postopératoire ou de la surveillance systématique à distance de la prise en charge chirurgicale lésionnelle initiale. Le praticien découvre alors une image ostéolytique qui peut signer une récidive lésionnelle, une cicatrisation osseuse hétérogène du lit osseux opéré ou l'apparition d'une lésion de caractéristique clinique et radiologique différente de la lésion initiale... ce qui nous replace dans les $1^{\text {re }}$ et $2^{\mathrm{e}}$ situations.

La pratique largement diffusée de la radiographie explique la fréquence avec laquelle on découvre inopinément une anomalie radioclaire des mâchoires. Le dossier radiographique établi dans le cadre d'un bilan orthopédique dento-maxillo-facial ou orthodontique en fournit un bel exemple. À l'inverse, il demeure fréquent que des avulsions successives ne soient pas encadrées par une radiographie globale préalable. À se focaliser sur un cliché rétroalvéolaire, le praticien sous-estime une image pathologique plus importante expliquant la mobilité d'une ou plusieurs dents.

Cliniquement, s'il existe une grande diversité de tumeurs, pseudo-tumeurs et kystes des mâchoires, leur symptomatologie d'appel est assez univoque.

Linterrogatoire bien conduit recensera le sexe, lâge du patient, ses antécédents personnels et familiaux, médicaux, chirurgicaux et allergiques : notion de « kystes des mâchoires » dans la famille, notion d'intervention chirurgicale stomatologique ou maxillofaciale pour dents de sagesse, « kyste », ou naevi cutanés. On demandera au patient s'il bénéficie d'un traitement anticoagulant ou antirésorbeur osseux de type bisphosphonates. Enfin, on demandera au patient ses doléances, le début des signes, l'intensité de la gêne physique ou douloureuse.

La tuméfaction reste le signe clinique dominant et souvent unique, pouvant occasionner une asymétrie du tiers supérieur (rare exophtalmie), du tiers moyen (prétragienne, génienne haute, labiale supérieure) ou du tiers inférieur facial (voussure latéralisée angulomandibulaire, génienne basse, jugale ou massétérine, ou mentonnière). Des signes inflammatoires des parties molles en regard (muqueuse, muscles, peau, conjonctive oculaire, muqueuse endonasale ou endosinusienne), bien souvent modérés, peuvent marquer la poussée évolutive révélatrice de la lésion (fig. 1). Une diplopie peut aussi en être révélatrice.

La tuméfaction osseuse correspondante est alors aisément reconnue, car elle fait corps avec l'os. Circonscrite, elle peut être vestibulaire, palatine ou linguale, maxillo-mandibulaire ou ramique (fig. 2). Sa palpation

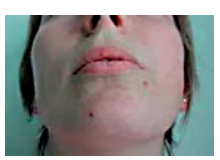

Fig. 1 :

Tuméfaction faciale du tiers inférieur gauche.

Fig. 2 :

Examen endobuccal : voussure du bord antérieur du ramus mandibulaire gauche.

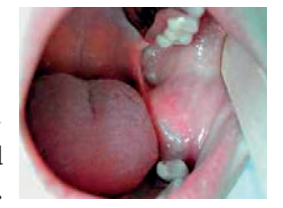


(bidigitale, endobuccale, exobuccale et endonarinaire) souligne son caractère ferme ou dépressible, et la possibilité d'un écoulement à la faveur d'une fistulisation. Dans d'autres cas, elle peut être plus discrète, soit du fait de son volume limité, soit du fait d'un développement axial centromédullaire ne soufflant pas les corticales donnant à la tuméfaction son caractère étendu, fusiforme, déformant un long segment mandibulaire, maxillaire voire l'ensemble d'un hémi-squelette facial. Les signes dentaires dans le secteur de la tumeur sont liés au développement du processus endosseux. Ils sont parfois inexistants : crête édentée en regard de la tuméfaction, dents présentes en bonne place, non mobiles, saines, bien traitées ou ne présentant pas de foyer infectieux évolutif, vivantes (répondant à la percussion ou au test au froid). Ailleurs, ils consistent en un foyer infectieux évident, une dyschromie de la couronne, des déplacements dentaires (malposition dentaire par égression, ingression, mésio-, disto-, linguo-, palato- ou vestibulo-version), des anomalies dévolution des dentitions (agénésie, dent incluse ou en désinclusion sous-muqueuse) ou encore une mobilité de la dent concernée ou des dents voisines. D’autres signes peuvent être associés : fistule muqueuse productive d'une sérosité claire ou puriforme, souvent au contact d'une dent, ulcération muqueuse et saignement de contact exceptionnel, retard de cicatrisation d'une alvéole dentaire après avulsion, séquestre osseux endobuccal mis à nu, adénopathie associée ou encore altération de la sensibilité cutanéo-muqueuse dans le territoire du nerf dentaire inférieur (trijumeau V3), voire sous-orbitaire (trijumeau V2).

Aussi, cet examen clinique incitera le praticien, dans la très grande majorité des cas, à faire réaliser un panoramique dentaire.

Au niveau de la mandibule, celui-ci garde aujourd'hui toute sa valeur dorientation diagnostique dans les lésions mandibulaires du fait de sa vision globale de l'arcade et de son caractère peu irradiant. Cependant, sa résolution modérée et son caractère tomographique $2 \mathrm{D}$ sous-estiment de façon notoire les atteintes dentaires, l'extension lésionnelle et surtout la présence d'éventuelles densités ou calcifications qui peuvent radicalement modifier l'orientation diagnostique.

Quant au maxillaire, même s'il est visible sur la même acquisition, les superpositions avec les structures naso-sinusales conduisent à une sous-estimation ou, au contraire, une sur-estimation lésionnelle qui doit orienter d'emblée vers la réalisation d'une tomodensitométrie ou scanner en coupes fines (TDM ou Dentascanner) ou mieux d'un CBCT (Cone Beam Computed Tomography).

Jusquà ces dernières années, la TDM permettait une analyse osseuse satisfaisante de ces lésions, mais sa résolution osseuse (en particulier face à des clichés long cône focalisés) était souvent insuffisante, en particulier dans l'analyse dentaire et en présence de matériel de traitement endocanalaire ou prothétique générant des artéfacts grevant l'analyse fine des images. Le CBCT s'est ainsi imposé rapidement. À petit ou grand champ, il permet au prix d'une irradiation nettement inférieure à celle de la TDM (rapport de 1 à 5 minimum en fonction des appareillages et des protocoles d'explorations radiologiques) l'obtention de coupes à très haute résolution osseuse, supérieure à celle de la TDM. Les artéfacts d’origine dentaire ou métallique sont beaucoup plus limités et, dans l'immense majorité des cas, ne gênent pas l'analyse dentaire fine. Les reconstructions et rétro-reconstructions peuvent permettre selon le champ initial d'acquisition l'obtention de pixels de moins de $100 \mu$, permettant du fait de leur caractère isomérique des reconstructions dans tous les plans de qualité nettement supérieure à celle du scanner.

Lanalyse dento-alvéolaire redevient dès lors la clé de voûte du diagnostic, essentielle dans ces lésions pour la plupart d’origine dentaire (odontogène).

En revanche, il faut bien garder à l'esprit que sa résolution en contraste très modérée ne permet pas l'analyse des tissus mous de façon satisfaisante, ce qui doit donc lui faire substituer la TDM dès lors qu'une injection de produit de contraste est nécessaire ; cela s’applique donc essentiellement au bilan des lésions malignes, celui des lésions odontogènes ne requérant pas d'injection. L'avènement des scanners "multi-énergie " viendra encore probablement modifier ces algorithmes d'exploration dans un proche avenir.

Beaucoup plus rarement, l'IRM pourra venir compléter le bilan.

\section{Rappels :}

Il faut à présent bannir la réalisation de certains clichés radiographiques peu performants devenus obsolètes; ils s'ajouteraient à d'autres clichés radiologiques complémentaires et ne feraient qu'accroître l'exposition du patient aux rayons (ex. : occlusal ou mordus dentaires, défilés mandibulaires, tomographies du ramus et de l'articulation temporo-mandibulaire).

\Ce bilan radiologique devra être orienté par une demande, rédigée par le clinicien, qui précisera avec soin les circonstances de découverte de la lésion, son siège, son caractère unique ou non, les hypothèses diagnostiques. L'examen radiologique, en particulier le CBCT, pourra alors être ciblé puisqu'il faut se souvenir que la résolution et donc la qualité de l’examen est d'autant meilleure que le champ d'exploration est réduit, sectorisé, optimisant en particulier l'analyse dento-alvéolaire et la recherche de structures denses. 


\section{ORIENTATION DIAGNOSTIQUE}

L'orientation diagnostique repose dès lors sur la corrélation précise des données cliniques et radiologiques: $\checkmark$ les données cliniques seront précisées, comme lâge et le sexe du patient (rareté de l'améloblastome chez l'enfant, rareté de l'odonto-améloblastome chez l'adulte, nette prédominance féminine, douleurs, bourgeonnement violacé muqueux et caractère délabrant des granulomes centraux à cellules géantes) ainsi que les antécédents (kyste essentiel du sujet jeune ayant bénéficié d'un traitement orthodontique) (fig. 3);

$\checkmark$ les données radiologiques souligneront l'aspect de lostéolyse, pure ou non, la présence éventuelle de radio-opacités au sein de l'image radioclaire, ses limites, la multiplicité des lésions, son siège, la présence d'une dent incluse, les rhizalyses...
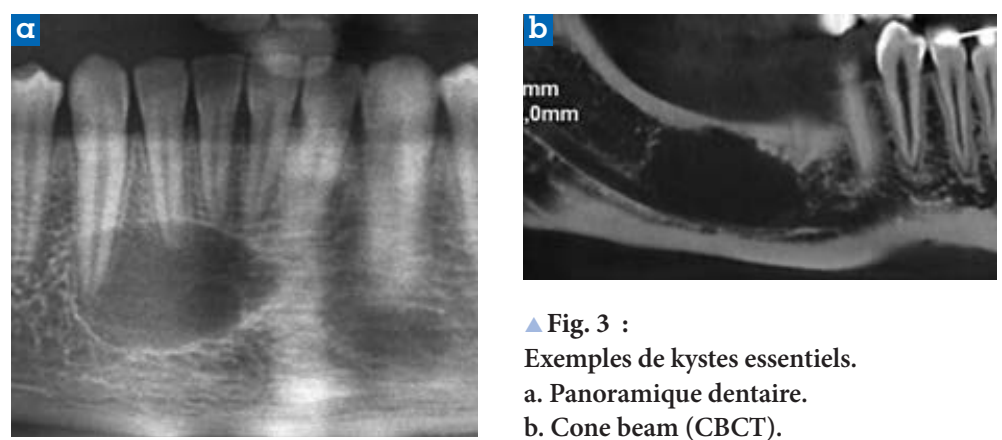

$\triangle$ Fig. 3 :

Exemples de kystes essentiels. a. Panoramique dentaire. b. Cone beam (CBCT).
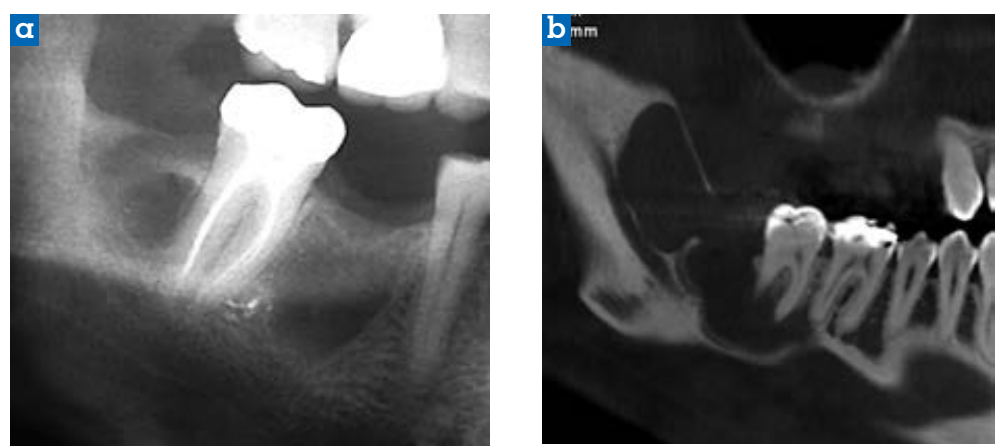

Fig. 4 :

Exemples de kératokystes.

a. Panoramique dentaire, b. Cone beam (CBCT)
L’analyse première soigneuse du panoramique dentaire est essentielle. Par sa vision globale des mâchoires, il montre d'abord le caractère unique ou multiple des lésions, précise leur siège et leur extension ; confronté aux données de l'interrogatoire et de l'examen clinique, il pourra ainsi avoir une première valeur d'orientation diagnostique, et va pouvoir guider la suite des explorations. Mais il faut garder à l'esprit qu'il reste insuffisant, avec des risques de faux positifs liés à sa technique tomographique 2D.

\section{DIFFÉRENTES IMAGES}

On pourra ainsi découvrir différentes images.

\section{Sous forme de géode}

Celle-ci se traduit, par une image au fond homogène, radioclaire voire hyperclaire (si le volume de la lésion est suffisant pour amincir les corticales), au contour net, finement souligné en périphérie d'un liseré dense. C'est, entre autres, l'image habituelle du kératokyste (fig. 4). En revanche, un contour "nuageux » peut traduire une poussée inflammatoire du processus en cause. Le contour peut aussi être épais, pouvant résulter de poussées inflammatoires successives.

Une lésion ostéolytique radioclaire à proximité d'une crête alvéolaire édentée doit orienter en premier lieu vers un kyste odontogène inflammatoire résiduel (fig. 5).

À l'inverse, si la géode est moins radiotransparente, mais grise, homogène ou non, il peut encore sagir d'une lésion kystique, close entre des corticales encore épaisses mais aussi d'une lésion compacte. Ainsi, mis à part le cas particulier du schwannome (tumeur compacte, mais très translucide aux rayons $\mathrm{X}$ ), un fibrome ossifiant par exemple, vu à un stade précoce, apparaît sous forme d'une géode au fond plus ou moins gris, très trompeur, la minéralisation de la composante cémentaire ou osseuse, caractéristique du fibrome, étant à peine ébauchée (fig. 6).

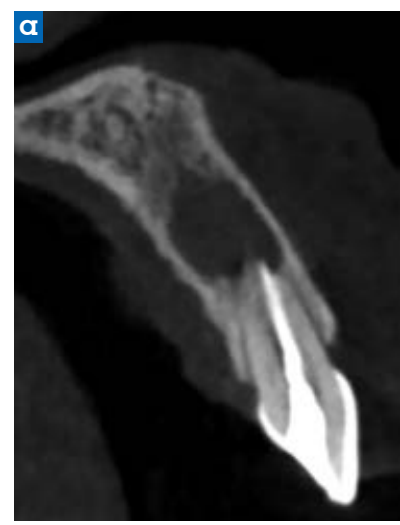

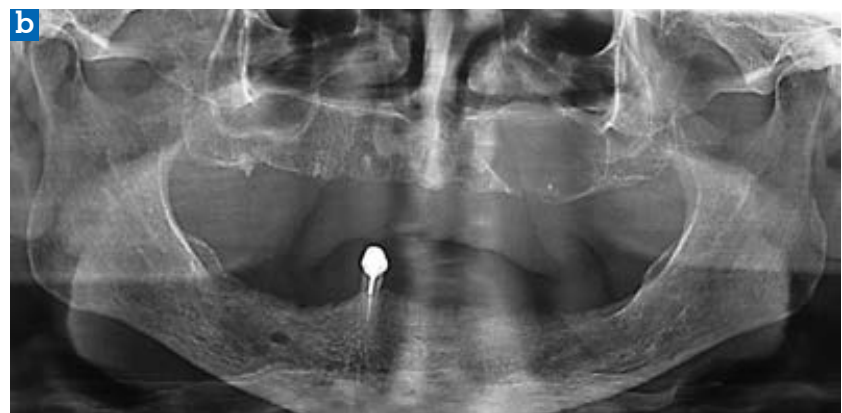

$\triangle$ Fig. 5 :

a. Kyste odontogène inflammatoire apical.

b. Kyste odontogène inflammatoire résiduel.

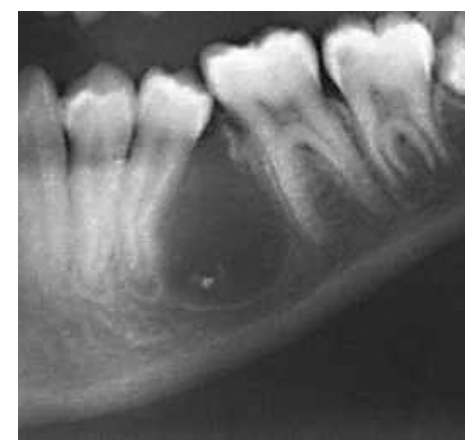

$\triangle$ Fig. 6 :

Fibrome ossifiant. 

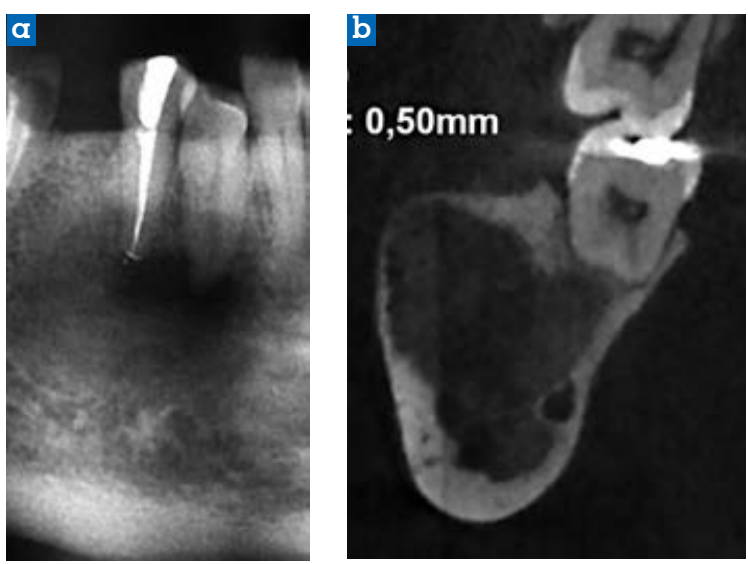

$\triangle$ Fig. 7 :

Exemples de fibromes cémento-ossifiants.

a. Panoramique dentaire, $b$. Cone beam (CBCT).
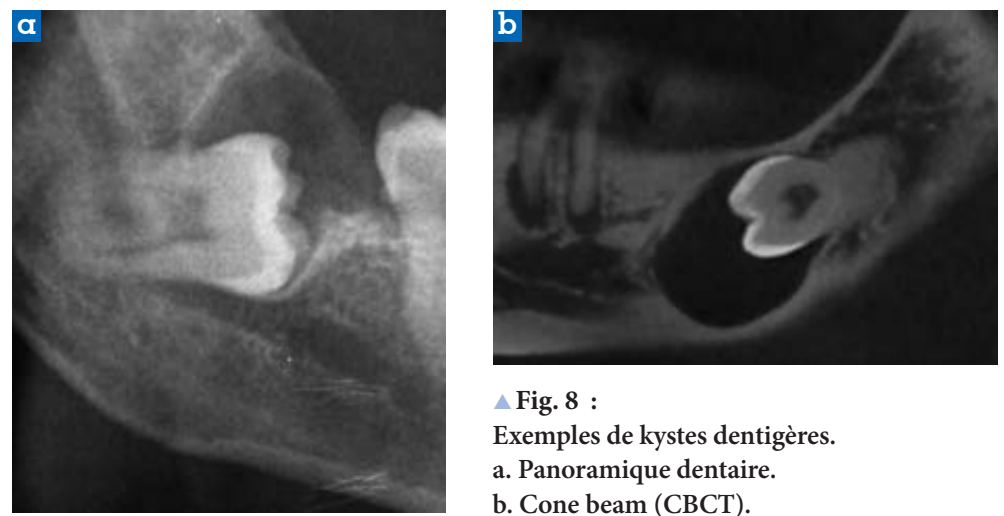

Fig. 8 :

Exemples de kystes dentigères.

a. Panoramique dentaire.

b. Cone beam (CBCT).

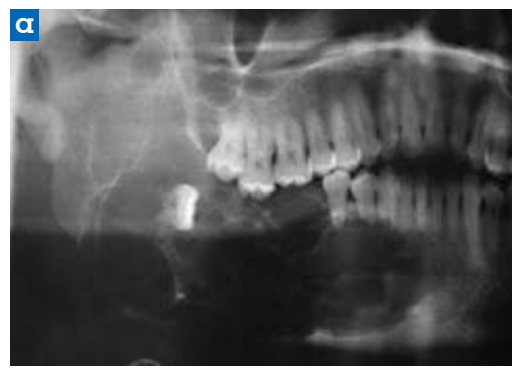

$\triangle$ Fig. 9 :

Exemples de rhizalyses dentaires dans des améloblastomes.

a. Panoramique dentaire.

b. Cone beam (СВCT).
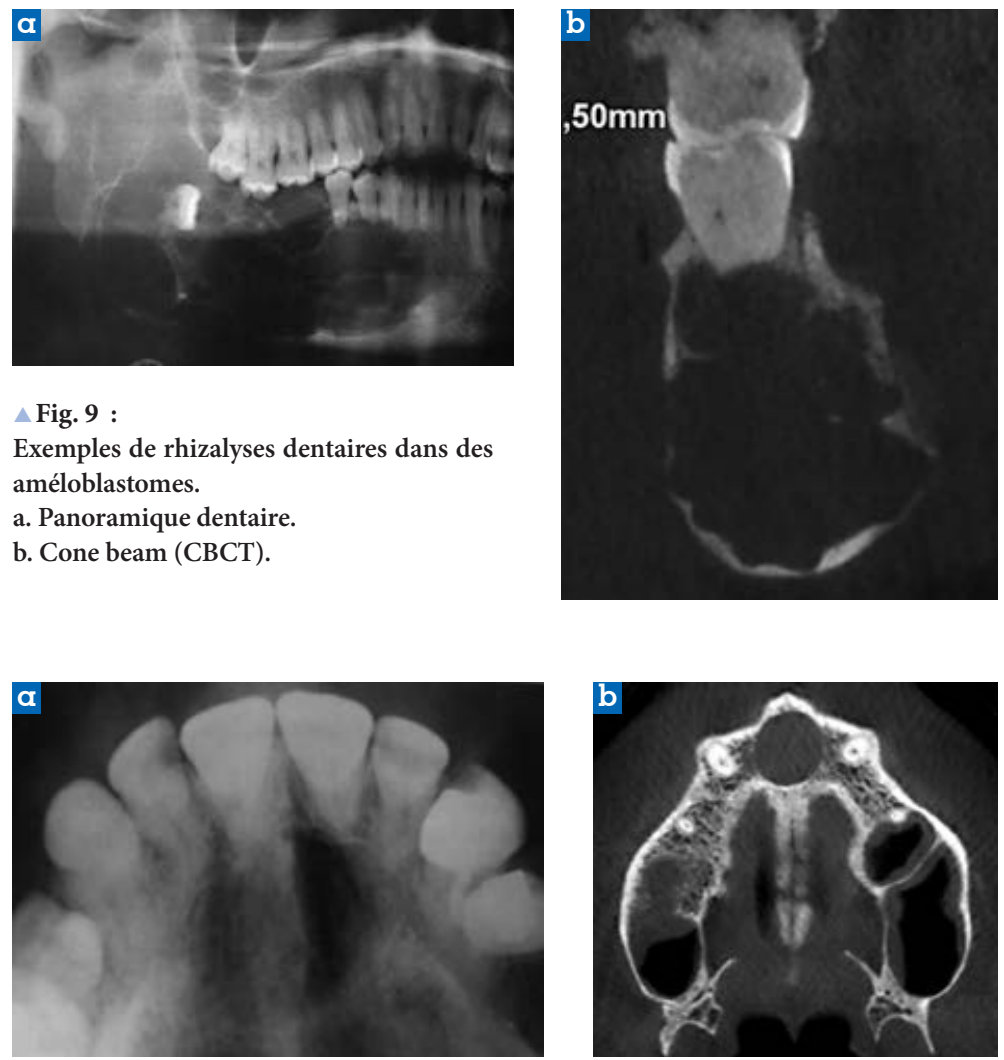

$\triangle$ Fig. 10 :

Exemples de kystes nasopalatins.

a. Cliché occlusal ou mordu, b. Cone beam (CBCT)

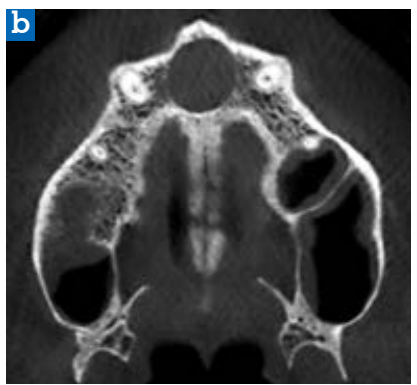

La confrontation avec les données cone beam CT va alors affiner le diagnostic. La résolution osseuse va permettre de confirmer le caractère lytique pur ou au contraire mettre en évidence de petites calcifications ou densités cémentaires possiblement passées inaperçues sur le panoramique (fig. 7) ; la résolution osseuse est en effet très supérieure à celle du panoramique dentaire; il faut cependant savoir que de telles composantes peuvent encore être sous-estimées car de taille infra-radiologique et ne seront mises en évidence que par l'examen histopathologique ; leur présence radiologique élimine par contre des hypothèses diagnostiques telles que améloblastome, kératokyste, kyste apico-dentaire ou dentigère... et oriente vers des hypothèses plus rares comme les lésions fibro-osseuses, d'origine mésenchymateuse ou autre.

Les hypothèses diagnostiques tiendront compte des éléments suivants.

I La présence d'une dent incluse : la tendance est grande, du fait de sa fréquence, d'associer à sa présence le diagnostic du fréquent kyste dentigère (anciennement folliculaire ou déruption) (fig. 8) ; mais elle est également très habituelle dans de nombreuses autres lésions odontogènes de type kératokyste, améloblastome, tumeur épithéliale odontogène calcifiante de Pindborg... et ne constitue donc aucunement un élément diagnostique spécifique.

I L'existence de rhizalyses : elles attestent d'une érosion des racines dentaires adjacentes à la lésion par son seul caractère érosif local (usure de chronicité ou d'agressivité) ; leur importance peut avoir une valeur d'orientation si elles sont majeures (comme dans un améloblastome si elles sont associées à ses autres caractéristiques diagnostiques) (fig. 9) ou au contraire si elles sont limitées ou absentes (éliminant ainsi l'améloblastome et orientant plutôt vers un kératokyste si elles sont limitées ou un kyste essentiel si elles sont absentes). Mais elles peuvent tout à fait sobserver au contact de communs mais volumineux kystes apico-dentaires, et ne signent à l'inverse en rien la malignité de la lésion même si des lyses extensives sont alors habituelles.

ILe siège de la lésion, non seulement maxillaire ou mandibulaire, mais aussi en portion dentée ou non (lésion odontogène ou non), symphysaire ou angulaire, incisivo-canin ou prémolo-molaire... Il peut être pathognomonique comme celui du kyste naso-palatin (géode bien bordée en arrière et située entre les racines des incisives centrales supérieures restées vivantes, plus ou moins divergentes en fonction de l'extension du kyste) (fig. 10). En fonction des antécédents, une lésion à distance de la portion dentée peut cependant aussi traduire une récidive polaire supérieure d'un kératokyste anciennement 


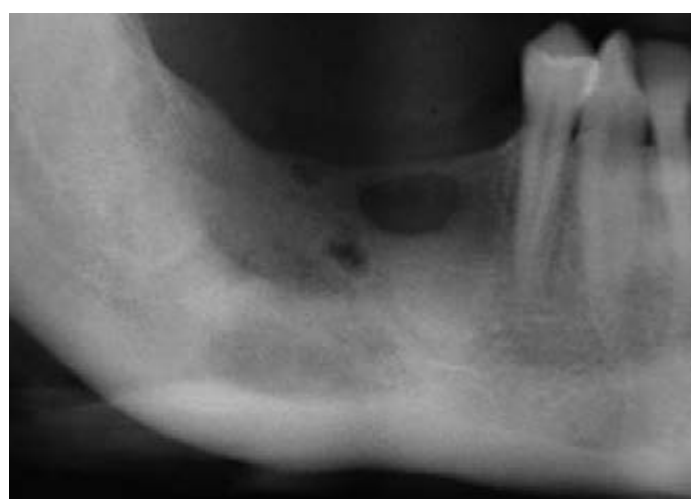

$\triangle$ Fig. 11 :

Récidive polaire supérieure d'un kératokyste opéré (panoramique dentaire).

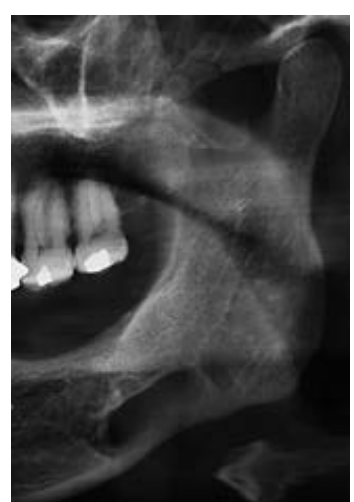

$\triangle$ Fig. 12 :

Lacune de Stafne (panoramique dentaire).

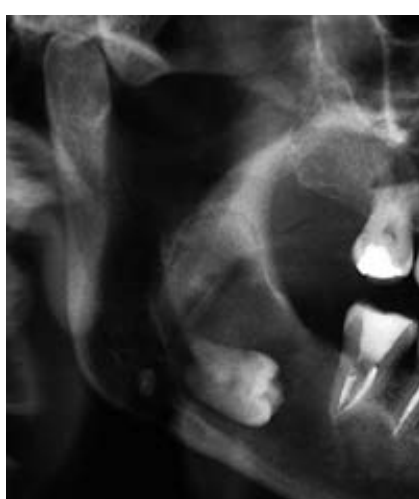

Fig. 13 :

Image atypique de kyste dentigère (panoramique dentaire).

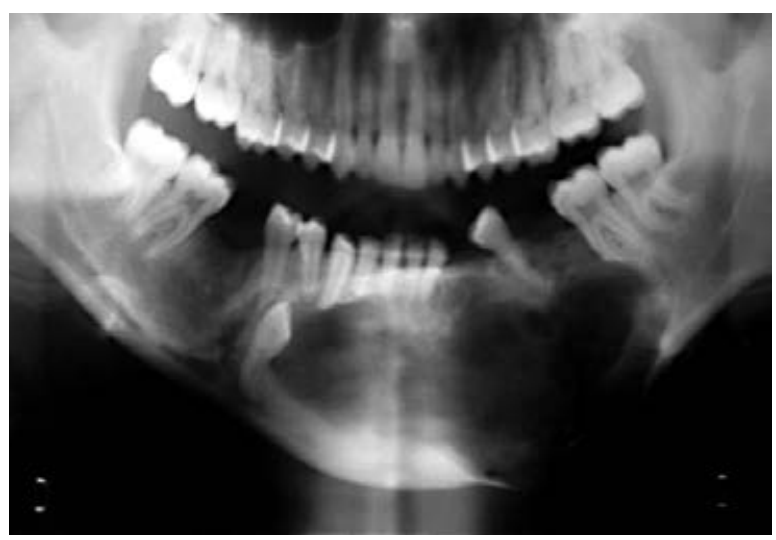

$\triangle$ Fig. 14 :

Améloblastome unikystique (panoramique dentaire).

opéré (fig. 11) ; ailleurs, il fait réfuter le diagnostic de lésion odontogène et évoquer une lacune de Stafne (image uni-géodique ronde ou ovalaire, au bord épais, très claire, située en frontière de l'angle mandibulaire et dans la portion basilaire de los) (fig. 12) ou encore une rare tumeur nerveuse du nerf dentaire inférieur.

\section{$\checkmark$ Le caractère uni- ou polygéodique :}

I La découverte d'une image monogéodique unique va orienter en priorité vers le fréquent kyste apico-dentaire s'il est bien limité et appendu à l'apex d'une dent mortifiée ou vers un kyste dentigère avec son rapport caractéristique vis-à-vis d'une dent incluse (attache du sac kystique au collet de la dent) (fig. 13). Ailleurs, l'extension dans l'axe de la mandibule, le siège angulo-maxillaire associé à des rhizalyses limitées feront plutôt évoquer une tumeur odontogénique kératokystique, alors qu'un aspect soufflant avec d'importantes rhizalyses fera penser au plus rare améloblastome dans sa forme unikystique trompeuse (fig. 14). À l'inverse, c'est l'absence de déviation dentaire et de rhizalyses malgré son volume, joint à l'aspect festonné interdentaire, qui orientera vers le kyste essentiel (fig. 15). Si le cone

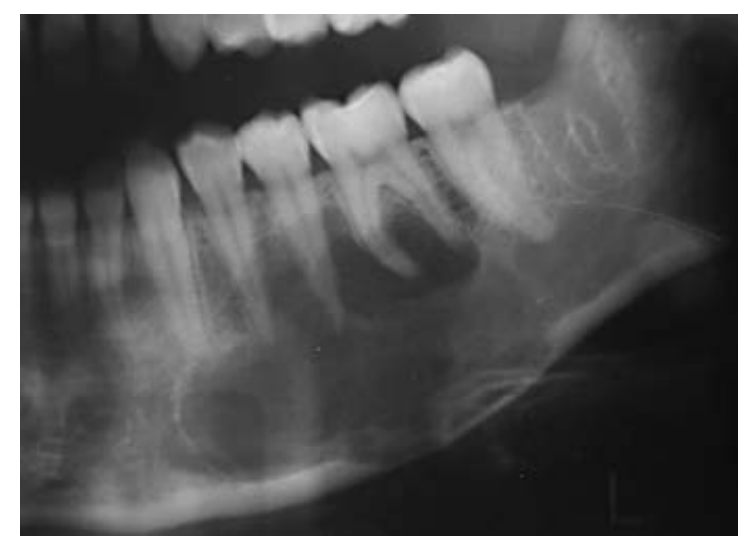

$\triangle$ Fig. 15 :

Kyste essentiel (panoramique dentaire).

beam est d'une grande sensibilité dans la détection fine de calcifications, comme celles observées dans la tumeur épithéliale odontogénique calcifiante de Pindborg... le diagnostic des lésions fibro-osseuses et assimilées dans leur forme précoce reste difficile ; elles simulent alors du fait de leur siège volontiers angulo-mandibulaire et de la fréquence des dents incluses associées, les lésions kystiques odontogènes beaucoup plus fréquentes; ce sont ces notions qui nous rappellent, du fait de la possibilité non rare des récidives dans ces pathologies, toute l'importance d'une histopathologie systématique, pour ne pas découvrir a posteriori des images de diagnostic et de traitement d'autant plus difficile que le diagnostic initial est méconnu. Rappelons aussi à cette occasion la possibilité de lésions intriquées comme la greffe d'un améloblastome au sein d'un banal kyste dentigère ; le pronostic reste alors à la lésion la plus agressive et donc dans ce cas à laméloblastome! Un siège plus atypique, comme en portion dentée incisivo-canine, maxillaire plutôt que mandibulaire, la corrélation avec lâge et le sexe du patient pourront également déboucher sur des lésions plus rares comme une tumeur odontogène 
adénomatoïde. Plus rarement, le siège spécifique suffit à évoquer la nature de la lésion, comme pour le kyste naso-palatin.

Des lésions multiples monogéodiques sont plus rares mais non exceptionnelles; leur analyse sémiologique est identique aux lésions uniques et, de même, un mauvais état bucco-dentaire fera évoquer en priorité de multiples foyers infectieux dentaires à l'origine de kystes odontogènes inflammatoires, avant d'évoquer le rare diagnostic de kératokystes multiples dans le cadre d'un syndrome de Gorlin-Goltz.
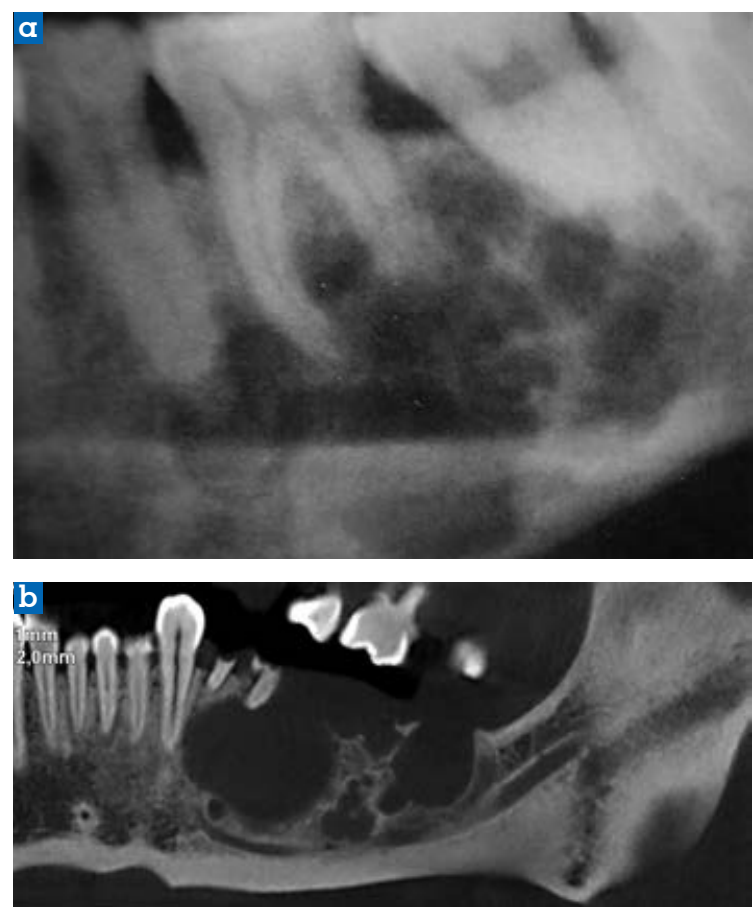

$\triangle$ Fig. 16 :

Exemples d'améloblastomes de forme radiologique multigéodique. a. Panoramique dentaire.

b. Cone beam (CBCT).

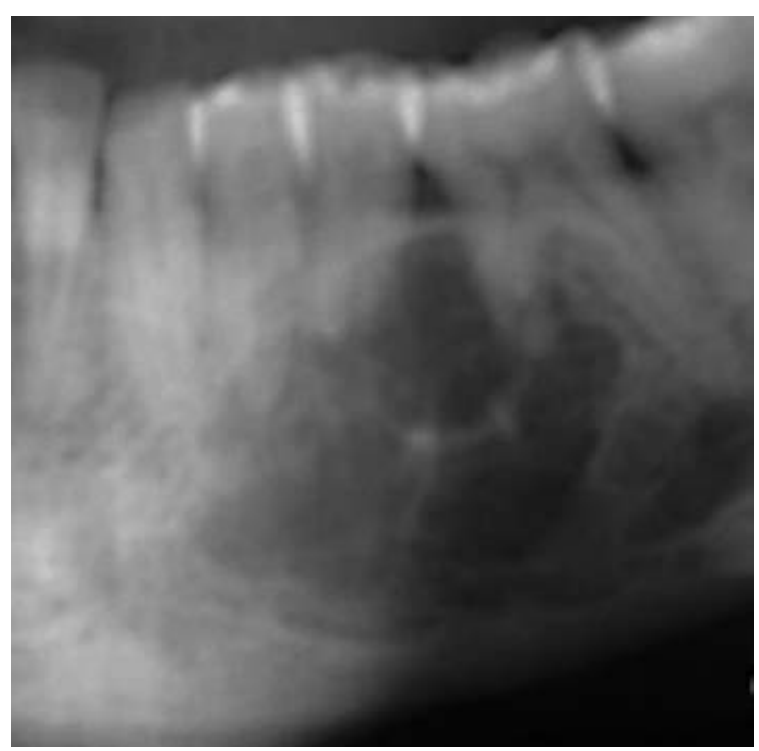

$\triangle$ Fig. 17 :

Granulome à cellules géantes.
ILe caractère polygéodique est souvent évocateur. Associé à des rhizalyses extensives, un aspect soufflant avec amincissement notable des corticales, il conduit naturellement au diagnostic d'améloblastome (fig. 16) ; beaucoup plus rarement à celui de tumeur odontogénique kératokystique sachant que, dans ce cas, l'aspect polygéodique est beaucoup plus rare et surtout moins marqué, y compris dans l'angle mandibulaire, localisation préférentielle de ces deux pathologies.

En revanche, une symptomatologie d'appel bruyante (signes inflammatoires nets) fait évoquer préférentiellement le diagnostic de kératokyste, d'autant plus probable qu'il est constaté au collet d'une molaire un écoulement puriforme ou d'apparence huileuse, parsemé de paillettes brillantes (de cholestérine ou kératine). De même, bien qu'inconstant, le contour épais de la géode est également en faveur de remaniements inflammatoires d'un kératokyste, aspect non observé avec les améloblastomes.

Ailleurs encore, des aspects dits " en nid d'abeille " (petites images polyédriques tassées les unes contre les autres) doivent aussi faire évoquer les non rares mais plus méconnus granulomes à cellules géantes (fig. 17). Un cloisonnement géométrique, à angle vif ou droit, peut aussi faire soupçonner le rare myxome ou un fibromyxome, au pronostic plus réservé du fait de son caractère infiltrant local en dehors de toute composante maligne associée.

\section{Sous forme de lacune}

Il s'agit également d'une lésion ostéolytique, mais qui, contrairement à la géode, définit mieux un espace tout simplement vide, une image d'ostéolyse sans frontière stricte. Celle-ci trouve toute son expression dans l'image classique du granulome éosinophile de l'histiocytose langherhansienne, où la résorption interdentaire aboutit à l'image classique de « dent flottante " (fig. 18). De façon plus agressive, on retrouve ici les tumeurs malignes (rarement primaire in situ, plus souvent secondaire métastatique ou par envahissement de proximité d'une lésion maligne muqueuse endobuccale); les contours sont alors irréguliers avec aspect mal limité entre l'os lysé et l'os normal.

\section{Sous forme de lésions mixtes}

Avec présence de calcifications ou de densités cémentaires intralésionnelles. Nous avons vu que la présence de ces dernières permet par exemple d'exclure des lésions de type kyste apicodentaire, kératokyste ou améloblastome...

Lorsque la radio-opacité de type dentaire constitue l'essentiel de l'image, il est aisé de conclure à un odontome, fréquent. Lopacité est habituellement cernée 

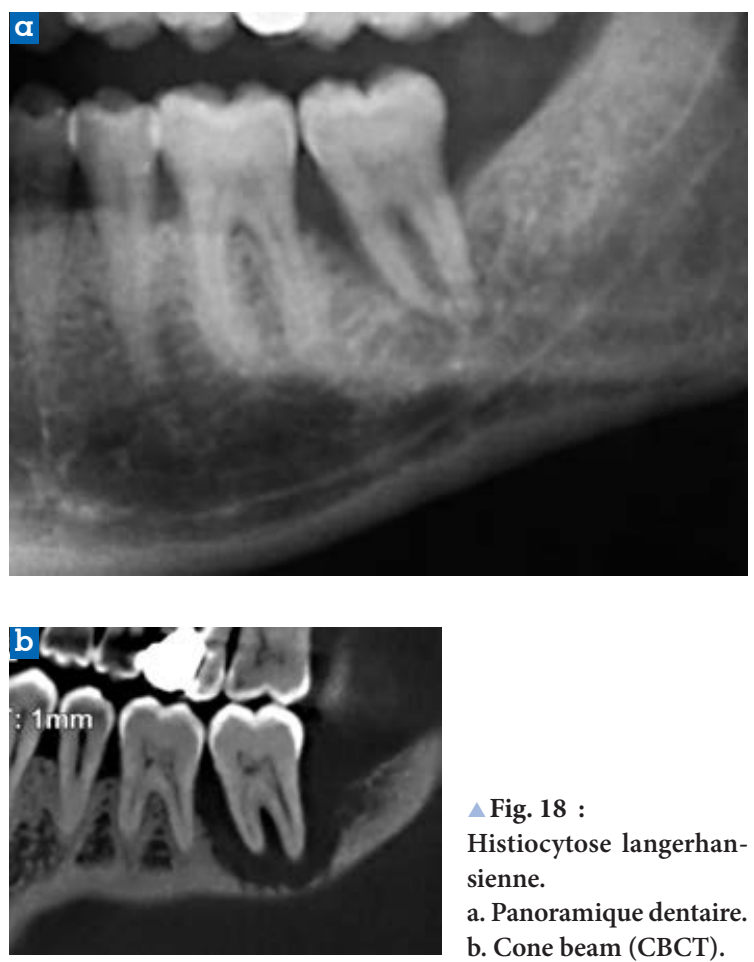

Fig. 18 :

Histiocytose langerhansienne.

a. Panoramique dentaire. b. Cone beam (CBCT).

d'un halo clair qui correspond à du tissu lésionnel non encore minéralisé. Ailleurs c'est le siège caractéristique péri-apical qui orientera vers le cémentoblastome.

Certaines lésions se traduisent aussi par des images polygéodiques, mais parsemées d’opacités fines, de type dentaire. Il s'agit ainsi de l'adéno-améloblastome, de la tumeur épithéliale odontogène calcifiée de Pindborg ou encore du kyste odontogène calcifié. Le fibro-odontome améloblastique, l'odonto-améloblastome, le fibrome odontogène pourront aussi être évoqués.

Les radio-opacités cémentaires sont reconnaissables par leur composante opaque, intermédiaire entre l’opacité dentaire et l'opacité osseuse.

Une des plus intéressantes lésions mixtes reste le fibrome cémento-ossifiant aux manifestations classiques de tumeur maxillaire et à la grande variabilité de présentation radiologique dominée par une opacité variable et surtout son caractère bien circonscrit.

Cette analyse lésionnelle globale corrélée aux données cliniques permet ainsi dans la très grande majorité des cas une orientation diagnostique précise. Ainsi, si la très grande majorité des lésions radioclaires correspond à des lésions kystiques de type apico-dentaire ou dentigère, toute discordance radioclinique sera discutée. La détection d’opacités réorientera vers des lésions de type odontogène mésenchymateuse, ailleurs de nature fibro-osseuse ou cémentaire. La multiplicité lésionnelle et l'aspect des lésions pourront au contraire orienter vers une maladie plus générale (syndrome de Gorlin, histiocytose langerhansienne...).
Un point essentiel reste cependant l'ultime confrontation «systématique » entre les données clinico-radiologiques et les conclusions anatomopathologiques. La possibilité de lésions intriquées, évolutives, source de récidives ultérieures, ne peut être évoquée que sur les aspects histologiques. À l'inverse, devant des lésions histologiquement aspécifiques (contenant des cellules géantes, ou épidermoïdes sans signe de kératinisation), le diagnostic histologique final reposera sur les données clinico-radiologiques, et sera rendu à la fine appréciation du stomatologue ou chirurgien maxillofacial spécialisé qui donnera à la lésion, son " épithète " final : chérubisme, granulome central à cellules géantes, kyste anévrysmal, tumeur brune de l'hyperparathyroïdisme, tumeurs à myéloplaxes pour une lésion à cellules géantes, "inflammatoire » ou " dentigère " pour un kyste épidermoïde sans signe de kératinisation).

\section{CONCLUSION}

Les lésions radioclaires des mâchoires sont fréquentes et le plus souvent de nature odontogène et bénigne. Mais leur fréquence recouvre aussi une très grande diversité lésionnelle, d’agressivité et de pronostic variables. D'autre part, d'autres affections non tumorales revêtent des aspects radiographiques superposables comme une infection qui peut présenter des aspects différents selon son stade évolutif ou son degré de maturation.

Ainsi une image radioclaire d'apparence banale peut cacher une lésion rare, odontogène ou non, ailleurs histologiquement intriquée, dont la non-reconnaissance initiale débouchera sur une surveillance non adaptée et de possibles récidives ultérieures au pronostic défavorable.

Dès lors, sur la base d'un interrogatoire le plus précis possible et d'un examen clinique rigoureux, la prise en charge du patient doit passer par une prise en charge multidisciplinaire ; celle-ci associera dans tous les cas une évaluation radiologique précise suivie, après intervention chirurgicale (au mieux par un stomatologue ou chirurgien maxillo-facial), d'un diagnostic anatomopathologique spécialisé à partir de la totalité de la pièce d'exérèse pour étiqueter correctement chaque processus et le situer dans la classification des tumeurs et pseudo-tumeurs établie, à juste titre, selon des critères anatomopathologiques. Les biopsies préopératoires ne sont plus pratiquées.

De cette prise en charge spécialisée rigoureuse dépendra la qualité de la surveillance des patients sachant que lavenir, pour nombre d'entre eux, est sous la dépendance d'un premier traitement chirurgical bien conduit. 


\section{Bibliographie}

[1] Martin-Duverneuil N, Auriol M. Les tumeurs maxillo-faciales. Imagerie, Anatomopathologie. Éditions Sauramps, 2004, 402 p.

[2] Martin-Duverneuil N. Tumeurs du massif facial. EMC Radiologie et imagerie médicale - musculosquelettique - neurologique maxillofaciale 31-675-A-20, 2013, $20 \mathrm{p}$.

[3] Barnes L, Eveson JW, Reichart P, Sidransky D (Eds). World health organization of tumours. Pathology and genetics of head and neck tumours. Lyon: IARC Press, 2005, p. 283-328.

[4] Martin-Duverneuil N, Hodez C. Les lacunes des maxillaires - Quelle vision avec le Cone Beam ? In : Hodez C, Bravetti P (eds) Imagerie dento-maxillo-faciale par faisceau conique " Cone Beam ». Montpellier: Sauramps, 2010, p. 213-220.

[5] Cavezian R, Pasquet G. Cone beam Imagerie diagnostique en odontostomatologie - Principes, résultats et perspectives. Issy-lesMoulineaux : Elsevier Masson, 2011.

[6] Ruhin B, Guilbert F, Bertrand J.-Ch. Traitement des kystes, tumeurs et pseudotumeurs bénignes des maxillaires. EMC Éditions Elsevier, Encyclopédie Médico Chirurgicale. Stomatologie/ Odontologie, 22-062-K10, 2005, 12 p.
[7] Guilbert F, Chomette G, Le Charpentier Y, Auriol M. Les tumeurs bénignes et pseudotumeurs des maxillaires. Rapport $d u$ XXXIIIe Congrès de Stomatologie et Chirurgie Maxillofaciale et Plastique de la Face. SeptOct 1993.

[8] Ruhin B, et al. Aggressive maxillary squamous odontogenic tumour in a child: histological dilemma and adaptative surgical behavior. Int J Oral Maxillofac Surg 2007;36(9):864-6.

[9] Pons Y, et al. Mandibular cuniculatum carcinoma: A propos of 3 cases and literature review. Head Neck 2012;34(2):291-5.

[10] Sioud S, et al. Giant cells tumors: three recurrent cases in young patients. Rev Stomatol Chir Maxillofac 2007;108(2):131-4.

[11] Ben Slama L, Ruhin B, Zoghbani A. Langerhans cell histiocytosis. Rev Stomatol Chir Maxillofac 2009;110(5):287-9.

[12] Ruhin B, Picard A, Martin-Duverneuil $\mathrm{N}$, Albertini AL, Goudot P. Les tumeurs odontogéniques strictement épithéliales kératokystiques : mise au point et revue générale. Rev Stomatol Chir Maxillofac 2011: 112(2):87-92.
[13] Steve M, Ernenwein D, Chaine A, Bertolus C, Goudot P, Ruhin-Poncet B. Ostéosarcomes des mâchoires : étude rétrospective de 14 patients pris en charge dans un Centre Hospitalier Universitaire français. Rev Stomatol Chir Maxillofac 2011;112(5):286-92.

[14] Ruhin-Poncet B, Bouattour A, Picard A, Menard P, Capron F, Bertrand J.Ch. Analyse rétrospective de 239 améloblastomes issus de 116 patients pris en charge dans un hôpital adulte français de 1994 à 2007. Rev Stomatol Chir Maxillofac 2011;112(5):269-79.

[15] Ruhin-Poncet B, Oprean N, MartinDuverneuil N, Capron F, Guilbert F, Bertrand JCh. Retrospective study of 349 odontogenic tumors in Pitié-Salpêtrière French Hospital from 1992 to 2007 and literature review. Rev Stomatol Chir Maxillofac (in press)

[16] Kadlub N, et al. Tumeurs kératokystiques de l'enfant et syndromes de Gorlin: comment expliquer les récidives et l'agressivité des lésions ? Rev Stomatol Chir Maxillofac 2012;113:148-154.

[16] Kadlub N, et al. Specificity of paediatric jawbone lesions: Tumours and pseudotumours. J Cranio Maxillofac Surg 2014;42(2):125-31.

\section{Cone Beam CT : imagerie diagnostique en odontostomatologie}

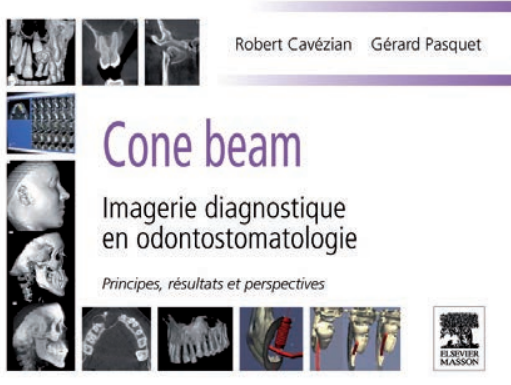

\section{Robert Cavézian, Gérard Pasquet}

Le Cone Beam est aujourd'hui la technique de référence en imagerie diagnostique dento-maxillofaciale. Remarquable par sa résolution d'image et la modestie des doses de rayons $\mathrm{X}$ délivrés, le Cone Beam s'impose dans tous les compartiments de la discipline. Cet ouvrage constitue ainsi un outil diagnostique incontournable pour tout radiologue, chirurgien-dentiste ou stomatologue désireux d'approfondir ses connaissances par une information claire, précise et exhaustive. 